\title{
A Unified Confidence Interval for Reliability-Related Quantities of Two-Parameter Weibull Distribution
}

\author{
Zhenlin Yang ${ }^{1}$, Min Xie ${ }^{2}$, Augustine C. M. Wong ${ }^{3}$ \\ ${ }^{1}$ School of Economics and Social Sciences, Singapore Management University \\ 90 Stamford Road, Singapore 178903. Email: zlyang@smu.edu.sg \\ ${ }^{2}$ Department of Industrial and System Engineering, National University of Singapore \\ Kent Ridge, Singapore 119260. Email: mxie@nus.edu.sg \\ ${ }^{3}$ SASIT, Atkinson Faculty of Liberal and Professional Studies, York University \\ North York, Ontario, M3J 1P3, Canada. Email: august@yorku.ca
}

April 5, 2006

\begin{abstract}
Statistical inference methods for the Weibull parameters and their functions usually depend on extensive tables, hence are rather inconvenient for the practical applications. In this paper, we propose a general method for constructing confidence intervals for the Weibull parameters and their functions, which eliminates the need for the extensive tables. The method is applied to obtain confidence intervals for the scale parameter, the mean-time-to-failure, the percentile function, and the reliability function. Monte Carlo simulation shows that these intervals possess excellent finite sample properties, having coverage probabilities very close to their nominal levels, irrespective of the sample size and the degree of censorship.
\end{abstract}

Keywords: Analytical adjustment; Confidence interval; Mean-time-to-failure; Percentile; Reliability; Type II censoring; Weibull-to-exponential transformation. 


\section{Introduction}

The Weibull distribution has been shown to be very useful in reliability and survival analyses. There are numerous papers and books dealing with various aspects of Weibull modeling, inference, as well as application. Some recent ones include Chen (1997), Heo et al. (2001), Jeng (2003), Murthy et al. (2003), and Yang and Xie (2003). It is well known that the existing statistical methods for inferences on the Weibull parameters and their functions usually depend on extensive tables obtained from Monte Carlo simulations (Bain and Engelhardt, 1991, Ch. 4), hence are rather inconvenient for the practical applications. Efforts on developing simple methods have been put in by, for example, Bain and Engelhardt (1981, 1986), and Chen (1997, 1998).

In this paper, we propose a general, nearly table-free method for constructing confidence intervals for the Weibull parameters and their functions, such as the scale parameter $(\alpha)$, the mean-time-to-failure (MTTF), the percentile function, and the reliability function. The method stems from the fact that a Weibull random variable raised to the power $\beta$ (the shape parameter) is an exponential random variable with mean $\alpha^{\beta}$. This transformation is often referred to as the Weibull-to-exponential (WTE) transformation (Keats et al., 2000; Xie et al., 2000). Exact inference methods exist for the exponential distribution. Therefore, when $\beta$ is known, an exact confidence interval (CI) for $\alpha^{\beta}$ can be obtained, which upon an inverse transformation gives an exact CI for $\alpha$. To construct an exact CI for a general monotonic function of $\alpha$ (indexed by $\beta$ ) such as the ones mentioned above, one simply applies this function to the lower and upper confidence limits for $\alpha$. Difficulty arises when $\beta$ is unknown. A common practice is to replace $\beta$ in the confidence limits by its estimator and treat the estimated $\beta$ as the true parameter value. The resulted $\mathrm{CI}$ is referred to in this article as the naive CI.

We show that the naive CI does not have a correct coverage, even when sample size is large. We derive a simple analytical adjustment to the naive CI, which gives an analytically adjusted naive (AAN) CI for the general function. The new AAN CI is simple, requiring only the conventional chi-squared table, and is asymptotically valid under mild conditions. However, its finite sample performance depends on the type of 
estimator used for $\beta$. Through a series of Monte Carlo experiments conducted on the CIs for $\alpha, \mathrm{MTTF}$, percentile and reliability, we find that the AAN CIs based on the nearly unbiased and efficient estimator of $\beta$ in Yang and Xie (2003) possess excellent finite sample properties, having coverage probabilities very close to their nominal levels, irrespective of the sample size and the degree of censorship. In contrast, the AAN CIs based on the (biased) maximum likelihood estimator may have coverage probabilities noticeably below their nominal levels. The Monte Carlo experiments further reveal that the naive CI often performs too poorly to be of any practical value, and that the AAN CI for $\alpha$ performs better than the corresponding CI given in Bain and Engelhardt (1986).

Section 2 introduces the new AAN method. Section 3 applies this method to obtain confidence intervals for various reliability-related Weibull quantities including the scale parameter, the MTTF, the percentile function and the reliability function. Section 4 presents Monte Carlo simulation results for the small sample performance of the intervals based on the AAN method. Also, the confidence interval for the Weibull scale parameter based on the AAN method is compared with that given in Bain and Engelhardt (1986) in terms of both the coverage probability and the average length of the intervals. Section 5 presents a real data example to compare the confidence intervals obtained by various methods. Section 6 provides some general discussions.

\section{The General Method}

A random variable $t$ is said to follow a two-parameter Weibull distribution if its probability density function has the form

$$
f(t ; \alpha, \beta)=\frac{\beta}{\alpha}\left(\frac{t}{\alpha}\right)^{\beta-1} \exp \left(-\left(\frac{t}{\alpha}\right)^{\beta}\right), \quad t>0,
$$

where $\alpha>0$ is the scale parameter and $\beta>0$ is the shape parameter. This distribution is very useful in reliability and survival analyses because of its flexibility: the failure rate function can be either increasing or decreasing depending on the value of the shape parameter $\beta$. The MTTF is $\mu=\alpha \Gamma(1+1 / \beta)$, the $100 p$ th percentile is $t_{p}=\alpha[-\log (1-$ $p)]^{1 / \beta}$, the mission reliability is $R(t)=\exp \left\{-(t / \alpha)^{\beta}\right\}$, and the failure rate is $h(t)=$ $\beta t^{\beta-1} / \alpha^{\beta}$. These quantities are of particular importance in reliability analysis. 


\subsection{The Weibull-to-exponential transformation}

It is well known that if $t \sim f(t ; \alpha, \beta)$, then the transformation $t^{\beta}$ follows an exponential distribution with mean $\alpha^{\beta}$. This transformation is generally referred to as the Weibull-to-exponential (WTE) transformation. Since statistical methods for analyzing the exponential distribution are simple and well developed (Nelson, 1982, p294), it makes the WTE transformation a potential approach for developing simple, general and yet accurate statistical inference methods for the Weibull distribution.

Consider a Type II censored life testing experiment where the test terminates at the $r$ th failure. Let $t_{1}, t_{2}, \ldots, t_{r}$ be the times at which the first $r$ failures occur and $n$ be the total number of items put on test. Define

$$
T(\beta)=2 \sum_{i=1}^{r}\left(\frac{t_{i}}{\alpha}\right)^{\beta}+2(n-r)\left(\frac{t_{r}}{\alpha}\right)^{\beta}=\frac{2 S(\beta)}{\alpha^{\beta}}
$$

where $S(\beta)=\sum_{i=1}^{r} t_{i}^{\beta}+(n-r) t_{r}^{\beta}$ is the total WTE-transformed testing time. Lawless (1982, p.103) showed that $T(\beta) \sim \chi_{2 r}^{2}$, a chi-squared distribution with $2 r$ degrees of freedom. When $\beta$ is known, the pivotal quantity $T(\beta)$ leads immediately to an exact $100(1-\delta) \%$ confidence interval (CI) for $\alpha^{\beta}$, which upon an inverse transformation gives an exact $100(1-\delta) \%$ CI for $\alpha$ :

$$
\left\{\left(\frac{2 S(\beta)}{\chi_{2 r, \delta / 2}^{2}}\right)^{1 / \beta},\left(\frac{2 S(\beta)}{\chi_{2 r, 1-\delta / 2}^{2}}\right)^{1 / \beta}\right\}=\left\{L_{\alpha}(\beta), U_{\alpha}(\beta)\right\} .
$$

where $\chi_{\nu, \delta}^{2}$ denotes the upper $\delta$-quantile of a $\chi_{\nu}^{2}$ random variable.

\subsection{The naive CI for a function of the Weibull parameters}

The distributional result for $T(\beta)$ and the interval (3) form the key to the new developments in this article. Now, consider a general function of the Weibull parameters, $\ell(\alpha, \beta)$, assumed to be strictly monotonic in $\alpha$, representing reliability-related quantities of interest such as the MTTF, the percentile function, and the reliability function. Without loss of generality, we assume that $\ell(\alpha, \beta)$ is strictly increasing in $\alpha$.

When $\beta$ is known, all the statistical inferences for $\ell(\alpha, \beta)$ can be carried out based on (2) and (3). Substituting the lower and upper confidence limits for $\alpha$ into the $\ell$ 
function, we have an exact $100(1-\delta) \%$ CI for $\ell(\alpha, \beta)$,

$$
\left\{\ell\left(L_{\alpha}(\beta), \beta\right), \quad \ell\left(U_{\alpha}(\beta), \beta\right)\right\}
$$

When $\beta$ is unknown, as it is often the case in the practical applications, a common practice is to replace $\beta$ in (4) by $\hat{\beta}$, an estimate of $\beta$, to give a CI for $\ell(\alpha, \beta)$,

$$
\left\{\ell\left(L_{\alpha}(\hat{\beta}), \hat{\beta}\right), \quad \ell\left(U_{\alpha}(\hat{\beta}), \hat{\beta}\right)\right\} .
$$

The interval given in (5) is referred to, in this article, as the naive CI for the function $\ell(\alpha, \beta)$. This procedure is very simple and hence should be attractive to the practitioners if it works well. Intuitively, this cannot possibly be true since the procedure ignores the variability in $\hat{\beta}$, an important quantity appearing on the exponent of almost every component in the lower and upper confidence limits. An intriguing question is that if (5) is not correct, can it be easily adjusted to give an interval that is correct at least when $n$ is large and approximates well when $n$ is small?

\subsection{The analytically adjusted naive $\mathbf{C I}$ for $\ell(\alpha, \beta)$}

Let $\ell^{-1}(\cdot, \beta)$ be the inverse of $\ell(\alpha, \beta)$ inverted in $\alpha$. Define

$$
W(\hat{\beta})=\frac{2 S(\hat{\beta})}{\left\{\ell^{-1}[\ell(\alpha, \beta), \hat{\beta}]\right\}^{\hat{\beta}}} .
$$

Clearly, $W(\hat{\beta})$ leads to $(5)$ when treated as a $\chi_{2 r}^{2}$ random variable. Thus, for the naive CI to be (asymptotically) valid, it is necessary that $W(\hat{\beta})$ is (asymptotically) equivalent to $T(\beta)$. The following arguments show that this is not true even when $n$ is large and hence an adjustment on $W(\hat{\beta})$ is necessary in order to use $\chi_{2 r}^{2}$ as a reference distribution.

Assume that $r / n \rightarrow p_{0}$ as $n \rightarrow \infty$ and that $\hat{\beta}$ is root- $n$ consistent. Write $W(\hat{\beta})=$ $T(\hat{\beta}) H(\hat{\beta})$, where $T(\hat{\beta})$ is defined by $(2)$ with $\beta$ replaced by $\hat{\beta}$, and

$$
H(\hat{\beta})=\left(\frac{\alpha}{\ell^{-1}[\ell(\alpha, \beta), \hat{\beta}]}\right)^{\hat{\beta}} .
$$

By a first-order Taylor expansion of $W(\hat{\beta})$ at $\hat{\beta}=\beta$ and using the fact that $H(\beta)=1$ and $W(\beta)=T(\beta)$, we have

$$
W(\hat{\beta})=T(\beta)+(\hat{\beta}-\beta)\left[T^{\prime}(\beta)+T(\beta) H^{\prime}(\beta)\right]+O_{p}(1),
$$


where $T^{\prime}(\beta)$ and $H^{\prime}(\beta)$ are, respectively, the first-order derivatives of $T(\hat{\beta})$ and $H(\hat{\beta})$ evaluated at $\hat{\beta}=\beta$. This immediately leads to

$$
W(\hat{\beta})=T(\beta)+2 \sqrt{n} Q(\beta)\left[k+p_{0} \beta H^{\prime}(\beta)\right]+O_{p}(1)
$$

where $Q(\beta)=\sqrt{n}[(\hat{\beta}-\beta) / \beta]$, and $k=\lim _{n \rightarrow \infty} \frac{1}{n} \mathrm{E}\left[\sum_{i=1}^{r} y_{i} \log y_{i}+(n-r) y_{r} \log y_{r}\right]$ with $y_{i}=\left(t_{i} / \alpha\right)^{\beta}$. Clearly, $k$ is a pure number depending only on the degree of censorship represented by $p_{0}$. If $\hat{\beta}$ is unbiased or the bias is of order $O\left(n^{-1}\right)$ or smaller, we have

$$
\mathrm{E}[W(\hat{\beta})]=\mathrm{E}(T(\beta)]+O(1)=2 r+O(1)
$$

This means that the first moment of $W(\hat{\beta})$ agrees with that of $T(\beta)$ up to at least second order, and hence for a fixed $n$, the means of the two quantities do not differ much.

Further, if $T(\beta)$ and $Q(\beta)$ are uncorrelated or weakly correlated in the sense that the correlation coefficient between the two quantities is of order $O\left(n^{-\frac{1}{2}}\right)$, we have

$$
\operatorname{Var}[W(\hat{\beta})]=4 r\left\{1+\tau^{2}\left[k+p_{0} \beta H^{\prime}(\beta)\right]^{2} / p_{0}\right\}+O(1)
$$

where $\tau^{2}=\lim _{n \rightarrow \infty} \operatorname{Var}[Q(\beta)]$, another pure number depending only on $p_{0}$. As $\operatorname{Var}[T(\beta)]$ $=4 r$, the result in (9) says that there exists a first-order difference between the second moments of $W(\hat{\beta})$ and $T(\beta)$. Hence the naive CI given in (5) is incorrect even when $n$ is large. For $(5)$ to have desirable properties, one has to adjust $W(\hat{\beta})$ so that at least the first two moments of $W(\hat{\beta})$ agree with those of $T(\beta)$. This can be done by first adjusting the variance of $W(\hat{\beta})$ using (9) and then recovering the changes on the mean. The adjusted pivotal quantity thus takes the following form:

$$
W^{*}(\hat{\beta})=\frac{W(\hat{\beta})+2 r[c(\hat{\alpha}, \hat{\beta})-1]}{c(\hat{\alpha}, \hat{\beta})},
$$

where the analytical adjustment factor $c(\alpha, \beta)$ has the following expression:

$$
c(\alpha, \beta)=\left\{1+\tau^{2}\left[k+p_{0} \beta H^{\prime}(\beta)\right]^{2} / p_{0}\right\}^{1 / 2},
$$

which depends on $p_{0}(\approx r / n)$ but may or may not depend on $\alpha$ and/or $\beta$. For certain $\ell(\alpha, \beta)$ functions that inference concern, $\beta H^{\prime}(\beta)$ vanishes or becomes a constant free of 
parameters (see Sections 3.1 and 3.3), and for other $\ell(\alpha, \beta)$ functions, $\beta H^{\prime}(\beta)$ becomes a function of $\beta$ or $\alpha$ or both (see Sections 3.2, 3.4 and 3.5).

Now, it is clear that the adjusted pivotal quantity $W^{*}(\hat{\beta})$ agrees with $T(\beta)$ in the first two moments when $n$ is large. It can further be shown that it is asymptotically equivalent to $T(\beta)$ by showing that the standardized statistics $\left[W^{*}(\hat{\beta})-2 r\right] /(2 \sqrt{r})$ and $[T(\beta)-2 r] /(2 \sqrt{r})$ both converge in distribution to the standard normal. This allows us to approximate the distribution of $W^{*}(\hat{\beta})$ by $\chi_{2 r}^{2}$, which leads to an analytically adjusted naive (AAN) CI for $\ell(\alpha, \beta)$ as:

$$
\left\{\ell\left[L_{\alpha}^{*}(\hat{\beta}), \hat{\beta}\right], \quad \ell\left[U_{\alpha}^{*}(\hat{\beta}), \hat{\beta}\right]\right\},
$$

where $L_{\alpha}^{*}(\hat{\beta})=2 S(\hat{\beta}) / \chi_{2 r, \delta / 2}^{2 *}$ and $U_{\alpha}^{*}(\hat{\beta})=2 S(\hat{\beta}) / \chi_{2 r, 1-\delta / 2}^{2 *}$, with $\chi_{2 r, \delta}^{2 *}=c(\hat{\alpha}, \hat{\beta}) \chi_{2 r, \delta}^{2}-$ $2 r[c(\hat{\alpha}, \hat{\beta})-1]$. Thus, adjusting the naive CI boils down to simply adjusting the chisquared cutoff values using the estimated analytical adjustment factor $c(\hat{\alpha}, \hat{\beta})$.

Now, we are left with the values of $\tau$ and $k$, and the question on degree of dependence between $T(\beta)$ and $Q(\beta)$. If $\hat{\beta}$ is the maximum likelihood estimator (MLE) or is asymptotically equivalent to the MLE, then $\tau$ is given in Bain and Engelhardt (1991, p.219). It is difficult to find the analytical expression for $k$. However, as it is a pure number, Monte Carlo simulation can be used to approximate its values.

Table 1 lists some values of $k$, each based on 10,000 samples of size 10,000. The values of $\tau^{2}$ given in Bain and Engelhardt (1991, p.219) are also incorporated into the table for the convenience of confidence interval construction. Other $k$ values for different $p_{0}$ can be well approximated by linear interpolations. When $p_{0}=1$, the true value for $k$ is $(1-$ Euler's constant $)=0.422784$, indicating the simulated $k$ value is very accurate. Table 1 also gives simulated values of the correlation coefficient $\rho$ between $T(\beta)$ and $Q(\beta)$, each based on 10,000 samples of size 10,000. The results indicate that the correlation between the two quantities are indeed weak when $n$ is large, confirming the validity of the variance calculation in (9). To check the stability of simulated $k$ values with respect to the sample size $n$, we have simulated the value of $k$ using various other sample sizes such as $n=20,000$. The simulated $k$ values change only in their 4 th decimal places when $n$ increases from 10,000 to 20,000 . 


\section{Table 1 here}

\subsection{Estimation of Weibull shape parameter}

One of the key factors affecting the finite sample performance of the AAN method is the estimation of the Weibull shape parameter $\beta$. There are several methods available in the literature for obtaining $\hat{\beta}$. Among them, the simplest ones may be the MLE (Lawless, 1982) and the modified MLE (MMLE) (Yang and Xie, 2003). From the general development given earlier, we see that it is important that $\hat{\beta}$ is unbiased. The MLE is known to be biased, especially when $n$ is small or censoring is heavy. The MMLE, however, is shown to be nearly unbiased and much more efficient than MLE when $n$ is small or censorship is heavy. The MMLE is asymptotically equivalent to MLE, thus the results in Table 1 are also applicable to the MMLE. The MLE solves $\beta$ from

$$
S_{p}(\beta)=\frac{r}{\beta}-\left(r \sum_{i=1}^{r} t_{i}^{\beta} \log t_{i}\right)\left(\sum_{i=1}^{r} t_{i}^{\beta}\right)^{-1}+\sum_{i=1}^{r} \log t_{i}=0,
$$

whereas the MMLE solves $\beta$ from

$$
S_{p}(\beta)=\frac{r-2}{\beta}-\left(r \sum_{i=1}^{r}{ }^{*} t_{i}^{\beta} \log t_{i}\right)\left(\sum_{i=1}^{r}{ }^{*} t_{i}^{\beta}\right)^{-1}+\sum_{i=1}^{r} \log t_{i}=0,
$$

where $\sum_{i=1}^{r}{ }^{*} w_{i}=\sum_{i=1}^{r} w_{i}+(n-r) w_{r}$. Comparing (12) and (13), we see that the modification is extremely simple. When needed, the corresponding estimator for $\alpha$, for a given $\hat{\beta}$, can be written as $\hat{\alpha}=\left(\frac{1}{r} \sum_{i=1}^{r}{ }^{*} t_{i}^{\hat{\beta}}\right)^{1 / \hat{\beta}}$.

\section{Applications of the AAN Method}

We now apply the general AAN method presented in Section 2 to obtain confidence intervals for the Weibull scale parameter, the MTTF, the percentile function and reliability function. We also highlight some other interesting cases where AAN method can be applied to show the generality of the method.

\subsection{CI for the scale parameter $\alpha$}

For inference concerning $\alpha, \ell(\alpha, \beta)=\alpha, H(\hat{\beta})=1, H^{\prime}(\beta)=0$, and $c(\alpha, \beta) \equiv c=$ $\left\{1+\tau^{2} k^{2} / p_{0}\right\}^{1 / 2}$ which depends only on the degree of censorship. Thus, an approximate 
$100(1-\delta) \%$ AAN CI for $\alpha$ based on $W^{*}(\hat{\beta})$ is given by

$$
\left\{\left(\frac{2 S(\hat{\beta})}{c \chi_{2 r, \delta / 2}^{2}-2 r(c-1)}\right)^{1 / \hat{\beta}}, \quad\left(\frac{2 S(\hat{\beta})}{c \chi_{2 r, 1-\delta / 2}^{2}-2 r(c-1)}\right)^{1 / \hat{\beta}}\right\} .
$$

\subsection{CI for the MTTF}

One of the aims of life testing is to determine the MTTF or to demonstrate that a certain MTTF is achieved with high confidence. In this case, we have $\ell(\alpha, \beta)=$ $\alpha \Gamma(1+1 / \beta), H(\hat{\beta})=[\Gamma(1+1 / \hat{\beta}) / \Gamma(1+1 / \beta)]^{\hat{\beta}}$, and $H^{\prime}(\beta)=-\beta^{-1} \psi(1+1 / \beta)$, where $\Gamma(\cdot)$ and $\psi(\cdot)$ are the gamma and digamma functions, respectively. Hence the AAN CI for the MTTF takes the form

$$
\left\{\Gamma\left(1+\frac{1}{\hat{\beta}}\right)\left(\frac{2 S(\hat{\beta})}{\chi_{2 r, \delta / 2}^{2 *}}\right)^{1 / \hat{\beta}}, \quad \Gamma\left(1+\frac{1}{\hat{\beta}}\right)\left(\frac{2 S(\hat{\beta})}{\chi_{2 r, 1-\delta / 2}^{2 *}}\right)^{1 / \hat{\beta}}\right\},
$$

where $\chi_{2 r, \delta}^{2 *}=c(\hat{\beta}) \chi_{2 r, \delta}^{2}-2 r[c(\hat{\beta})-1]$ and $c(\hat{\beta})=\left\{1+\tau^{2}\left[k-p_{0} \psi(1+1 / \hat{\beta})\right]^{2} / p_{0}\right\}^{1 / 2}$. In this case, the analytical adjustment factor depends on the shape parameter.

\subsection{CI for the percentile function}

The $100 p$ th percentile of the Weibull distribution is $t_{p}=\alpha[-\log (1-p)]^{1 / \beta}$. In this case, we have $\ell(\alpha, \beta)=\alpha[-\log (1-p)]^{1 / \beta}, H(\hat{\beta})=[-\log (1-p)]^{1-\hat{\beta} / \beta}$, and $H^{\prime}(\beta)=$ $-\beta^{-1} \log [-\log (1-p)]$. The AAN CI for $t_{p}$ is

$$
\left\{\left(-\frac{2 S(\hat{\beta})}{\chi_{2 r, \delta / 2}^{2 *}} \log (1-p)\right)^{1 / \hat{\beta}},\left(-\frac{2 S(\hat{\beta})}{\chi_{2 r, 1-\delta / 2}^{2 *}} \log (1-p)\right)^{1 / \hat{\beta}}\right\}
$$

where $\chi_{2 r, \delta}^{2 *}=c \chi_{2 r, \delta}^{2}-2 r(c-1)$ and $c^{2}=1+\tau^{2}\left\{k-p_{0} \log [-\log (1-p)]\right\}^{2} / p_{0}$. Like the case of confidence interval for $\alpha$, the analytical adjustment factor $c$ is again a scalar depending only on the degree of censorship.

\subsection{CI for the reliability function}

For the reliability function $R(t)$, we have $\ell(\alpha, \beta)=\exp \left\{-(t / \alpha)^{\beta}\right\}, H(\hat{\beta})=(t / \alpha)^{\beta-\hat{\beta}}$, and $H^{\prime}(\beta)=-\log (t / \alpha)$. The AAN CI for $R(t)$ is

$$
\left\{\exp \left(-\frac{t^{\hat{\beta}} \chi_{2 r, \delta / 2}^{2 *}}{2 S(\hat{\beta})}\right), \exp \left(-\frac{t^{\hat{\beta}} \chi_{2 r, 1-\delta / 2}^{2 *}}{2 S(\hat{\beta})}\right)\right\}
$$


where $\chi_{2 r, \delta}^{2 *}=c(\hat{\alpha}, \hat{\beta}) \chi_{2 r, \delta}^{2}-2 r[c(\hat{\alpha}, \hat{\beta})-1]$ and $c^{2}(\hat{\alpha}, \hat{\beta})=1+\tau^{2}\left[k-p_{0} \hat{\beta} \log (t / \hat{\alpha})\right]^{2} / p_{0}$. The analytical adjustment factor depends on both the scale and shape parameters which need to be estimated. MLE, MMLE or other consistent estimators can be used. However, MMLE is seen to be preferred from Monte Carlo simulation.

\subsection{CIs for other reliability-related quantities}

Confidence intervals for other reliability-related quantities can be constructed in a similar way. It is just a matter of specifying the function $\ell(\alpha, \beta)$ and finding the related quantities. For example, if the quantity of interest is the hazard rate, we have $\ell(\alpha, \beta)=\beta t^{\beta-1} / \alpha^{\beta}$; if the quantity of interest is the standard deviation, we have $\ell(\alpha, \beta)=\alpha\left[\Gamma(1+2 / \beta)-\Gamma^{2}(1+1 / \beta)\right]^{1 / 2}$; and if the quantity of interest is the $j$ th moment, we have $\ell(\alpha, \beta)=\alpha^{j} \Gamma(1+j / \beta)$.

\section{Monte Carlo Simulation}

The simplicity and the flexibility of the new AAN method is clearly demonstrated in the earlier section. It is, however, necessary to investigate its properties when the sample size is not large or when the censorship is heavy. In this section, we conduct simulation studies to compare the three confidence intervals discussed in this article:

(a) the naive confidence interval,

(b) the AAN confidence interval using MLE of $\beta$, and

(c) the AAN confidence interval using MMLE of $\beta$.

The comparisons are based on the coverage probabilities of the three CIs. Also in this section, a comparison is made on the average lengths of the proposed CI for $\alpha$ and the corresponding CI given in Bain and Engelhardt (1986). All simulations are carried out using FORTRAN 90. The IMSL/RNWIB subroutine is used for generating the Weibull random numbers. Each simulated coverage probability is based on 10,000 samples. Since the coverage is independent on the choice of $\alpha$ value, without loss of generality, $\alpha$ is set to be 1 in all simulation. Further, as the behavior of the intervals depends very little on the value of $\beta$, we only report the results that correspond to $\beta=2$. More detailed simulation results are available from the first author upon request. 
Table 2 summarizes the simulated coverage probabilities of the CIs for $\alpha$. From the table, we observe that (i) the AAN CI using the MMLE of $\beta$ performs exceptionally well, even when the sample size $n$ is small and the censorship is heavy (represented by a small value $p_{0}$ ); (ii) the AAN CI using the MLE of $\beta$ can perform poorly when $n$ and/or $p_{0}$ is small, showing that it is important to use a less biased and more efficient estimator of $\beta$, such as the MMLE of Yang and Xie (2003), when constructing CIs for the Weibull scale parameter; and (iii) the naive CI has a rather poor coverage, which deteriorates with the increase of the degree of censorship. Simulation results (not reported for brevity) show that the performance of the CIs depends very little on the actual value of $\beta$.

Table 2 here

Table 3 records the simulated coverage probabilities of the three CIs for the percentile function. Various percentile points are considered. Again the simulation results show that the AAN CI using the MMLE of $\beta$ is the best, followed by the AAN CI using the MLE of $\beta$, and the naive CI. Using the MMLE of $\beta$ instead of the MLE improves the coverage of the AAN CI noticeably, particularly in the cases of small percentiles with heavy censorship. The naive CI for percentile often performs too poorly to be of any practical value. The $95 \%$ and $99 \%$ AAN CIs using the MMLE of $\beta$ for the low percentiles, such as the 10th percentile, are slightly liberal, but still far better than the other two CIs. Simulation for other percentiles, as well as under different $\beta$ values and sample sizes, has also been carried out. The results (not reported for brevity) are consistent with those in Table 3.

\section{Table 3 here}

Table 4 summaries the simulation results for the MTTF. In this case, the analytical adjustment factor depends on the parameter $\beta$ that has to be estimated. Simulation results show that this estimation does not affect much on the relative performance of the three CIs. Once again, the AAN CI using the MMLE of $\beta$ outperforms the other two CIs, especially when $n$ is small. It is interesting to note that, when the censorship is light $\left(p_{0}>0.6\right.$, say), the AAN CI using the MLE of $\beta$ perform no better than the naive 
CI. Once again, simulation results (not reported for brevity) show that the coverage probabilities of the CIs depend very little on the actual value of $\beta$.

\section{Table 4 here}

Table 5 presents the simulated coverage probabilities of the confidence bounds for the reliability function $R(t)$. In this case, the analytical adjustment factor depends on both $\alpha$ and $\beta$. From Table 5 we see that the AAN CI using the MMLE of $\beta$ still outperforms the AAN CI using the MLE of $\beta$ in general. The coverage of the AAN CI using the MLE of $\beta$ varies a lot with the value of $R(t)$, especially in the case of the $90 \%$ CI where the reported the coverage probability changes from 0.8401 to 0.9474 as $R(t)$ changes from 0.05 to 0.95 . Finally, the naive CI can give an extremely poor coverage of $R(t)$, much below its nominal level.

\section{Table 5 here}

Table 6 compares the coverage probabilities and the average lengths of the CIs for $\alpha$, based on the proposed AAN method and the method presented in Bain and Engelhardt (1986). The results show that our method provides a better interval in terms of both the coverage probability and the average length of the confidence interval. The interval given by Bain and Engelhardt (1986) can perform poorly when sample size is small or the data are heavily censored. In contrast, our intervals perform well in all the situations, especially the interval using the MMLE of $\beta$.

\section{Table 6 here}

\section{An Illustrative Example}

We now consider a real data example to illustrate the use of the WTE transformationbased AAN CIs developed in this paper and to compare them with the CI obtained using the third-order approximation method proposed by Wong and $\mathrm{Wu}$ (2000). We use the data reported in Lawless (1975, p.258). The data contain 40 observations, but only the first 28 are actually observed, which are in log scale: 


$$
\begin{array}{rrrrrrr}
-2.982 & -2.849 & -2.546 & -2.350 & -1.983 & -1.492 & -1.443 \\
-1.394 & -1.386 & -1.269 & -1.195 & -1.174 & -0.845 & -0.620 \\
-0.576 & -0.548 & -0.247 & -0.195 & -0.056 & -0.013 & 0.006 \\
0.033 & 0.037 & 0.046 & 0.084 & 0.221 & 0.245 & 0.296
\end{array}
$$

We compute CIs for the following five quantities: the scale parameter, the MTTF, the 10th percentile, the median, and the 90th percentile. The MLEs for the shape parameter and the five quantities of interest are, respectively, 1.0984, 1.1692, 1.1287, 0.1507, 0.8375, and 2.4984. The corresponding MMLEs are, respectively, 1.0363, 1.1775, 1.1606, 0.1342, 0.8267, and 2.6332. We note that the MLE and MMLE of $\beta$ do differ noticeably, thus causing the noticeable differences between the MLEs and MMLEs of the other quantities of interest.

Table 7 summarizes the computed confidence intervals. From the table, we observe that among the three CIs discussed in this paper, the naive CI is always the shortest and often significantly shorter than the other two, followed by the AAN CI using the MLE of $\beta$ and the AAN CI using the MMLE of $\beta$. In line with the simulation results reported in the previous section, we conclude that the AAN CI using the MMLE of $\beta$ is the most reliable one. Also from Table 7, we see that the MMLE-based AAN CIs for the scale parameter, the MTTF and the median are comparable with those based on Wong and Wu's method, though slightly shorter. However, when inference concerns the 10th and 90th percentiles (or low and high percentiles in general), our method and Wong and Wu's method produce quite different confidence intervals.

Table 7 here

\section{Discussion}

A general method, called analytically adjusted naive (AAN) method, is proposed for constructing confidence intervals for the reliability-related Weibull quantities. The AAN method is rather general and is exemplified in this paper for the cases of the scale parameter, MTTF, percentile and reliability. Excellent performance of the AAN CI is observed, in particular the one based on the MMLE of $\beta$.

The AAN method has potential extensions to more complicated inference problems often occured in, for example, the two-sample comparisons and the Weibull regressions. 
It also has potential extensions to the more complicated censoring schemes such as the Type II progressive censoring (Balakrishnan and Basu, 1995, p.25). Generally speaking, the AAN method is applicable whenever exact methods of inference are available for the WTE-transformed observations.

The main attractiveness of the proposed method lies in its simplicity and accuracy as compared with the methods available in the literature. Computing the estimate of the shape parameter is the only part that involves numerical iteration.

The proposed method does not have a straightforward generalization to Type I censored data. However, as Nelson (1982, p.249) commented, many people analyze Type I data as if it were Type II data and this is often satisfactory in practice. Also, recently, Jeng and Meeker (2000) studied procedures for computing confidence intervals for parameters or quantiles based on Type I censored data from a location-scale family. Wong and Wu's (2000) procedure is applicable to Type I data from a location-scale family as well. Doganaksoy and Schmee (2000) gave a general discussion on the papers of Jeng and Meeker and Wong and $\mathrm{Wu}$, where the simplicity of a methodology seems to have been stressed on. The proposed method can be implemented into popular commercial software without any technical difficulty.

\section{Acknowledgements}

We thank a referee for the constructive and detailed comments that have led to significant improvements on the earlier versions of this paper. Zhenlin Yang's research is supported by the Wharton-SMU research center, Singapore Management University. Augustine C. M. Wong's research is supported by NSERC, Canada. 


\section{References}

Bain, L. J. and Engelhardt, M. (1981). Simple approximate distributional results for confidence and tolerance limits for the Weibull distribution based on maximum likelihood estimators. Technometrics 23, 15-20.

Bain, L. J. and Engelhardt, M. (1986). Approximate distributional results based on maximum likelihood estimators for the Weibull distribution. Journal of Quality Technology 18, 174-181.

Bain, L. J. and Engelhardt, M. (1991). Statistical Analysis of Reliability and Life-Testing Models. Marcel Dekker Inc., New York.

Balakrishnan, N. and Basu, A. P. (1995). The Exponential Distribution - Theory, Methods and Applications. Gordon and Breach, Amsterdam.

Chen, Z. (1997). Statistical inference about the shape parameter of the Weibull distribution. Statistics and Probability Letters 36, 85-90.

Chen, Z. (1998). Joint estimation for the parameters of Weibull distribution. Journal of Statistical Planning and Inference 66, 113-120.

Doganaksoy, N. and Schmee, J. (2000). Practical aspects of corrected likelihood ratio confidence intervals: a discussion of Jeng-Meeker and Wong-Wu. Technometrics 42, 156-159.

Heo, J. H., Salas, J. D. and Kim, K. D. (2001). Estimation of confidence intervals of quantiles for the Weibull distribution. Stochastic Environmental Research Risk Assessment 15, 284-309.

Jeng, S. L. (2003). Exact sample size determination in a Weibull test plan when there is time censoring. Journal of Statistical Computation and Simulation 73, 389-408.

Jeng, S. L. and Meeker, W. Q. (2000). Comparisons of approximate confidence interval procedures for Type I censored data. Technometrics 42, 135-148.

Keats, J. B., Nahar, P. C. and Korbell, K. M. (2000). A study of the effect of misspecification of the Weibull shape parameter on confidence bounds based on the 
Weibull-exponential transformation. Quality and Reliability Engineering International 16: 27-31.

Lawless, J. F. (1975). Construction of tolerance bounds for the Extreme Value and Weibull distributions. Technometrics 17, 255-261.

Lawless, J. F. (1982). Statistical Models and Methods for Lifetime Data. Wiley, New York.

Murthy, D. N. P., Xie, M. and Jiang, R. Y. (2003). Weibull Models. Wiley, New York.

Nelson, W. (1982). Applied Life Data Analysis. Wiley, New York.

Wong, A. C. M. and Wu, J. (2000). Practical small-sample asymptotics for distributions used in life-data analysis. Technometrics 42, 149-155.

Xie, M., Yang, Z. L. and Gaudoin, O. (2000). More on the mis-specification of the shape parameter with Weibull-to-exponential transformation. Quality and Reliability Engineering International 16, 281-290.

Yang, Z. L. and Xie, M. (2003). Efficient estimation of the Weibull shape parameter based on a modified profile likelihood. Journal of Statistical Computation and Simulation 73, 115-123. 
Table 1. A Summary of the Constant Values

\begin{tabular}{rrrr}
\hline$p_{0}$ & $\tau^{2}$ & $k$ & $\rho$ \\
\hline 1.0 & 0.607927 & 0.422642 & -0.021417 \\
0.9 & 0.767044 & 0.206893 & -0.007954 \\
0.8 & 0.928191 & 0.042456 & -0.012622 \\
0.7 & 1.122447 & -0.090239 & -0.012683 \\
0.6 & 1.372781 & -0.195195 & -0.030083 \\
0.5 & 1.716182 & -0.272584 & -0.008047 \\
0.4 & 2.224740 & -0.320973 & -0.014092 \\
0.3 & 3.065515 & -0.336481 & -0.011844 \\
0.2 & 4.738764 & -0.311191 & -0.001159 \\
0.1 & 9.744662 & -0.227626 & 0.000345 \\
\hline
\end{tabular}

Table 2. Simulated Coverage Probabilities for Confidence Intervals for $\alpha$ :

(a) Naive CI using MLE of $\beta$; (b) AAN CI using MLE; (c) AAN CI using MMLE.

\begin{tabular}{rr|rrr|rrr|rrr}
\hline & & \multicolumn{3}{|c|}{$90 \%$ CI } & \multicolumn{3}{c|}{$95 \%$ CI } & \multicolumn{3}{c}{$99 \%$ CI } \\
\cline { 3 - 10 }$n$ & $p_{0}$ & (a) & $(\mathrm{b})$ & $(\mathrm{c})$ & (a) & (b) & (c) & (a) & $(\mathrm{b})$ & $(\mathrm{c})$ \\
\hline 20 & 1.0 & 0.8544 & 0.8748 & 0.9005 & 0.9133 & 0.9278 & 0.9443 & 0.9684 & 0.9751 & 0.9840 \\
& 0.9 & 0.8581 & 0.8638 & 0.8939 & 0.9141 & 0.9199 & 0.9422 & 0.9704 & 0.9727 & 0.9817 \\
& 0.8 & 0.8567 & 0.8567 & 0.8921 & 0.9149 & 0.9149 & 0.9412 & 0.9703 & 0.9704 & 0.9819 \\
& 0.7 & 0.8491 & 0.8512 & 0.8904 & 0.9039 & 0.9058 & 0.9348 & 0.9627 & 0.9636 & 0.9787 \\
& 0.6 & 0.8321 & 0.8491 & 0.8939 & 0.8881 & 0.9022 & 0.9368 & 0.9504 & 0.9597 & 0.9783 \\
& 0.5 & 0.7900 & 0.8402 & 0.8955 & 0.8568 & 0.8959 & 0.9421 & 0.9266 & 0.9574 & 0.9801 \\
& 0.4 & 0.7235 & 0.8353 & 0.9016 & 0.7957 & 0.8987 & 0.9513 & 0.8842 & 0.9637 & 0.9880 \\
\hline 50 & 1.0 & 0.8672 & 0.8866 & 0.8972 & 0.9259 & 0.9387 & 0.9465 & 0.9806 & 0.9862 & 0.9894 \\
& 0.9 & 0.8790 & 0.8859 & 0.8973 & 0.9335 & 0.9386 & 0.9471 & 0.9809 & 0.9827 & 0.9858 \\
& 0.8 & 0.8862 & 0.8863 & 0.8996 & 0.9381 & 0.9382 & 0.9477 & 0.9847 & 0.9847 & 0.9883 \\
& 0.7 & 0.8774 & 0.8793 & 0.8933 & 0.9287 & 0.9299 & 0.9412 & 0.9795 & 0.9801 & 0.9841 \\
& 0.6 & 0.8697 & 0.8834 & 0.8999 & 0.9216 & 0.9321 & 0.9469 & 0.9776 & 0.9816 & 0.9873 \\
& 0.5 & 0.8333 & 0.8780 & 0.9017 & 0.8945 & 0.9315 & 0.9484 & 0.9612 & 0.9776 & 0.9862 \\
& 0.4 & 0.7795 & 0.8783 & 0.9043 & 0.8521 & 0.9330 & 0.9529 & 0.9366 & 0.9809 & 0.9896 \\
& 0.3 & 0.6969 & 0.8744 & 0.8965 & 0.7731 & 0.9306 & 0.9516 & 0.8741 & 0.9826 & 0.9917 \\
\hline
\end{tabular}


Table 3. Simulated Coverage Probabilities for Percentile Limits: $n=50$,

(a) Naive CI using MLE of $\beta$; (b) AAN CI using MLE; (c) AAN CI using MMLE.

\begin{tabular}{|c|c|c|c|c|c|c|c|c|c|c|}
\hline \multirow{2}{*}{\multicolumn{2}{|c|}{$p_{0}$}} & \multicolumn{3}{|c|}{$90 \% \mathrm{CI}$} & \multicolumn{3}{|c|}{$95 \%$ CI } & \multicolumn{3}{|c|}{$99 \% \mathrm{CI}$} \\
\hline & & (a) & (b) & (c) & (a) & (b) & (c) & (a) & (b) & (c) \\
\hline \multirow[t]{9}{*}{$t_{0.10}$} & 1.0 & 0.5146 & 0.8615 & 0.8941 & 0.5900 & 0.8999 & 0.9308 & 0.7205 & 0.9482 & 0.9660 \\
\hline & 0.9 & 0.5279 & 0.8645 & 0.9032 & 0.6029 & 0.9021 & 0.9337 & 0.7302 & 0.9453 & 0.9675 \\
\hline & 0.8 & 0.5420 & 0.8609 & 0.9034 & 0.6177 & 0.8962 & 0.9313 & 0.7476 & 0.9412 & 0.9641 \\
\hline & 0.7 & 0.5524 & 0.8553 & 0.9032 & 0.6329 & 0.8917 & 0.9334 & 0.7564 & 0.9385 & 0.9610 \\
\hline & 0.6 & 0.5660 & 0.8416 & 0.8994 & 0.6520 & 0.8793 & 0.9301 & 0.7713 & 0.9315 & 0.9595 \\
\hline & 0.5 & 0.6051 & 0.8423 & 0.9060 & 0.6828 & 0.8828 & 0.9327 & 0.7978 & 0.9293 & 0.9620 \\
\hline & 0.4 & 0.6445 & 0.8296 & 0.8992 & 0.7178 & 0.8666 & 0.9253 & 0.8183 & 0.9158 & 0.9553 \\
\hline & 0.3 & 0.6998 & 0.8283 & 0.9051 & 0.7681 & 0.8662 & 0.9326 & 0.8519 & 0.9158 & 0.9594 \\
\hline & 0.2 & 0.7484 & 0.8144 & 0.9025 & 0.8123 & 0.8602 & 0.9347 & 0.8836 & 0.9140 & 0.9627 \\
\hline \multirow[t]{9}{*}{$\overline{t_{0.25}}$} & 1.0 & 0.6755 & 0.8771 & 0.8976 & 0.7615 & 0.9189 & 0.9383 & 0.8659 & 0.9623 & 0.9748 \\
\hline & 0.9 & 0.6849 & 0.8750 & 0.9022 & 0.7681 & 0.9204 & 0.9413 & 0.8755 & 0.9616 & 0.9748 \\
\hline & 0.8 & 0.7100 & 0.8772 & 0.9031 & 0.7920 & 0.9192 & 0.9458 & 0.8917 & 0.9626 & 0.9764 \\
\hline & 0.7 & 0.7411 & 0.8726 & 0.9062 & 0.8142 & 0.9195 & 0.9466 & 0.9091 & 0.9630 & 0.9776 \\
\hline & 0.6 & 0.7778 & 0.8745 & 0.9086 & 0.8494 & 0.9210 & 0.9473 & 0.9257 & 0.9645 & 0.9798 \\
\hline & 0.5 & 0.8055 & 0.8703 & 0.9024 & 0.8704 & 0.9175 & 0.9436 & 0.9376 & 0.9622 & 0.9778 \\
\hline & 0.4 & 0.8264 & 0.8545 & 0.8947 & 0.8874 & 0.9104 & 0.9390 & 0.9519 & 0.9615 & 0.9790 \\
\hline & 0.3 & 0.8502 & 0.8528 & 0.8954 & 0.9042 & 0.9062 & 0.9377 & 0.9596 & 0.9603 & 0.9777 \\
\hline & 0.2 & 0.8190 & 0.8359 & 0.9010 & 0.8780 & 0.8949 & 0.9428 & 0.9453 & 0.9544 & 0.9785 \\
\hline \multirow[t]{8}{*}{$t_{0.5}$} & 1.0 & 0.8275 & 0.8890 & 0.9044 & 0.8953 & 0.9405 & 0.9518 & 0.9650 & 0.9815 & 0.9855 \\
\hline & 0.9 & 0.8483 & 0.8859 & 0.9030 & 0.9075 & 0.9371 & 0.9497 & 0.9692 & 0.9810 & 0.9870 \\
\hline & 0.8 & 0.8583 & 0.8814 & 0.8995 & 0.9193 & 0.9339 & 0.9452 & 0.9730 & 0.9794 & 0.9848 \\
\hline & 0.7 & 0.8720 & 0.8803 & 0.8997 & 0.9289 & 0.9341 & 0.9464 & 0.9765 & 0.9791 & 0.9845 \\
\hline & 0.6 & 0.8772 & 0.8773 & 0.8951 & 0.9303 & 0.9307 & 0.9442 & 0.9803 & 0.9806 & 0.9863 \\
\hline & 0.5 & 0.8716 & 0.8763 & 0.8983 & 0.9249 & 0.9292 & 0.9454 & 0.9778 & 0.9791 & 0.9857 \\
\hline & 0.4 & 0.8407 & 0.8718 & 0.8973 & 0.8996 & 0.9235 & 0.9454 & 0.9585 & 0.9736 & 0.9830 \\
\hline & 0.3 & 0.7696 & 0.8627 & 0.8995 & 0.8418 & 0.9176 & 0.9476 & 0.9215 & 0.9710 & 0.9855 \\
\hline \multirow[t]{8}{*}{$t_{0.75}$} & 1.0 & 0.8895 & 0.8909 & 0.8990 & 0.9417 & 0.9421 & 0.9475 & 0.9847 & 0.9850 & 0.9868 \\
\hline & 0.9 & 0.8868 & 0.8882 & 0.8980 & 0.9380 & 0.9387 & 0.9453 & 0.9820 & 0.9828 & 0.9854 \\
\hline & 0.8 & 0.8724 & 0.8818 & 0.8937 & 0.9237 & 0.9323 & 0.9412 & 0.9783 & 0.9817 & 0.9863 \\
\hline & 0.7 & 0.8537 & 0.8849 & 0.8978 & 0.9130 & 0.9340 & 0.9455 & 0.9689 & 0.9802 & 0.9857 \\
\hline & 0.6 & 31 & 0.8812 & 0.8979 & 0.8875 & 0.9324 & 0.9460 & 0.9548 & 0.9797 & 0.9870 \\
\hline & 0.5 & 0.7730 & 0.8789 & 0.8978 & 0.8453 & 0.9326 & 0.9502 & 0.9287 & 0.9811 & 0.9884 \\
\hline & 0.4 & 0.7089 & 0.8873 & 0.9017 & 0.7899 & 0.9384 & 0.9547 & 0.8933 & 0.9839 & 0.9919 \\
\hline & 0.3 & 0.6262 & 0.8940 & 0.9000 & 0.7057 & 0.9502 & 0.9584 & 0.8235 & 0.9962 & 0.9968 \\
\hline \multirow[t]{7}{*}{$\overline{t_{0.95}}$} & 1.0 & 0.8399 & 0.8868 & 0.8936 & 0.9039 & 0.9399 & 0.9484 & 0.9690 & 0.9858 & 0.9891 \\
\hline & 0.9 & 0.7999 & 0.8861 & 0.8962 & 0.8706 & 0.9395 & 0.9482 & 0.9499 & 0.9849 & 0.9892 \\
\hline & 0.8 & 0.7665 & 0.8950 & 0.9030 & 0.8442 & 0.9429 & 0.9517 & 0.9307 & 0.9835 & 0.9884 \\
\hline & 0.7 & 0.7170 & 0.8926 & 0.9025 & 0.8002 & 0.9456 & 0.9513 & 0.9027 & 0.9878 & 0.9921 \\
\hline & 0.6 & 0.6652 & 0.8928 & 0.8974 & 0.7479 & 0.9456 & 0.9509 & 0.8679 & 0.9893 & 0.9926 \\
\hline & 0.5 & 0.6172 & 0.9036 & 0.9026 & 0.6964 & 0.9579 & 0.9573 & 0.8231 & 0.9947 & 0.9945 \\
\hline & 0.4 & 0.5535 & 0.9098 & 0.8983 & 0.6344 & 0.9654 & 0.9539 & 0.7610 & 0.9983 & 0.9930 \\
\hline
\end{tabular}


Table 4. Simulated Coverage Probabilities for Confidence Intervals for MTTF:

(a) Naive CI using MLE of $\beta$; (b) AAN CI using MLE; (c) AAN CI using MMLE.

\begin{tabular}{ll|ccc|ccc|ccc}
\hline & & \multicolumn{3}{|c|}{$90 \%$ CI } & \multicolumn{3}{c|}{$95 \%$ CI } & \multicolumn{3}{c}{$99 \%$ CI } \\
\cline { 3 - 11 }$n$ & $p_{0}(\mathrm{a})$ & $(\mathrm{b})$ & $(\mathrm{c})$ & $(\mathrm{a})$ & $(\mathrm{b})$ & $(\mathrm{c})$ & $(\mathrm{a})$ & $(\mathrm{b})$ & $(\mathrm{c})$ \\
\hline 10 & 1.0 & 0.8274 & 0.8526 & 0.9002 & 0.8881 & 0.9059 & 0.9404 & 0.9473 & 0.9586 & 0.9813 \\
& 0.9 & 0.8258 & 0.8371 & 0.8906 & 0.8794 & 0.8895 & 0.9325 & 0.9436 & 0.9510 & 0.9761 \\
& 0.8 & 0.8196 & 0.8242 & 0.8900 & 0.8776 & 0.8823 & 0.9317 & 0.9401 & 0.9440 & 0.9737 \\
& 0.7 & 0.8039 & 0.8067 & 0.8878 & 0.8639 & 0.8659 & 0.9279 & 0.9311 & 0.9323 & 0.9712 \\
\hline 20 & 1.0 & 0.8570 & 0.8752 & 0.8979 & 0.9111 & 0.9257 & 0.9458 & 0.9690 & 0.9772 & 0.9854 \\
& 0.9 & 0.8521 & 0.8595 & 0.8881 & 0.9074 & 0.9150 & 0.9375 & 0.9658 & 0.9698 & 0.9809 \\
& 0.8 & 0.8590 & 0.8619 & 0.8944 & 0.9164 & 0.9177 & 0.9394 & 0.9686 & 0.9696 & 0.9796 \\
& 0.7 & 0.8507 & 0.8528 & 0.8890 & 0.9059 & 0.9080 & 0.9366 & 0.9668 & 0.9672 & 0.9793 \\
& 0.6 & 0.8415 & 0.8532 & 0.8988 & 0.8981 & 0.9066 & 0.9435 & 0.9593 & 0.9643 & 0.9795 \\
& 0.5 & 0.7930 & 0.8340 & 0.8962 & 0.8607 & 0.8934 & 0.9412 & 0.9346 & 0.9534 & 0.9786 \\
\hline 50 & 1.0 & 0.8726 & 0.8880 & 0.8986 & 0.9267 & 0.9385 & 0.9461 & 0.9778 & 0.9822 & 0.9861 \\
& 0.9 & 0.8818 & 0.8884 & 0.8993 & 0.9321 & 0.9351 & 0.9432 & 0.9784 & 0.9816 & 0.9866 \\
& 0.8 & 0.8809 & 0.8820 & 0.8957 & 0.9364 & 0.9371 & 0.9455 & 0.9834 & 0.9836 & 0.9866 \\
& 0.7 & 0.8744 & 0.8768 & 0.8918 & 0.9271 & 0.9291 & 0.9395 & 0.9799 & 0.9806 & 0.9867 \\
& 0.6 & 0.8617 & 0.8776 & 0.8966 & 0.9196 & 0.9314 & 0.9456 & 0.9761 & 0.9803 & 0.9876 \\
& 0.5 & 0.8309 & 0.8748 & 0.8969 & 0.8919 & 0.9275 & 0.9463 & 0.9614 & 0.9763 & 0.9858 \\
0.4 & 0.7801 & 0.8734 & 0.8999 & 0.8526 & 0.9222 & 0.9497 & 0.9322 & 0.9730 & 0.9862 \\
\hline
\end{tabular}


Table 5. Simulated Coverage Probabilities for Reliability Bounds: $n=50$,

(a) Naive CI using MLE of $\beta$; (b) AAN CI using MLE; (c) AAN CI using MMLE.

\begin{tabular}{|c|c|c|c|c|c|c|c|c|c|c|}
\hline \multirow[b]{2}{*}{$R(t)$} & \multirow[b]{2}{*}{$p_{0}$} & \multicolumn{3}{|c|}{$90 \%$ CI } & \multicolumn{3}{|c|}{$95 \%$ CI } & \multicolumn{3}{|c|}{$99 \%$ CI } \\
\hline & & (a) & (b) & (c) & (a) & (b) & (c) & (a) & (b) & (c) \\
\hline \multirow[t]{6}{*}{0.05} & 1.0 & 0.4325 & 0.8690 & 0.8936 & 0.5023 & 0.9166 & 0.9389 & 0.6277 & 0.9623 & 0.9739 \\
\hline & 0.9 & 0.4310 & 0.8592 & 0.8876 & 0.5012 & 0.9054 & 0.9343 & 0.6228 & 0.9546 & 0.9714 \\
\hline & 0.8 & 0.4405 & 0.8571 & 0.8897 & 0.5122 & 0.9066 & 0.9331 & 0.6380 & 0.9531 & 0.9716 \\
\hline & 0.7 & 0.4427 & 0.8523 & 0.8857 & 0.5203 & 0.8994 & 0.9313 & 0.6415 & 0.9519 & 0.9730 \\
\hline & 0.6 & 0.4629 & 0.8488 & 0.8891 & 0.5366 & 0.8995 & 0.9338 & 0.6613 & 0.9486 & 0.9709 \\
\hline & 0.5 & 0.4815 & 0.8401 & 0.8798 & 0.5564 & 0.8873 & 0.9271 & 0.6799 & 0.9409 & 0.9673 \\
\hline \multirow[t]{6}{*}{0.25} & 1.0 & 0.6726 & 0.8793 & 0.8929 & 0.7542 & 0.9351 & 0.9433 & 0.8612 & 0.9810 & 0.9864 \\
\hline & 0.9 & 47 & 0.8772 & 0.8909 & 0.7700 & 0.9322 & 0.9424 & 0.8743 & 0.9801 & 0.9859 \\
\hline & 0.8 & 0.7177 & 0.8774 & 0.8896 & 0.7912 & 0.9298 & 0.9411 & 0.8884 & 0.9784 & 0.9857 \\
\hline & 0.7 & 0.7407 & 0.8776 & 0.8894 & 0.8149 & 0.9327 & 0.9442 & 0.9055 & 0.9800 & 0.9863 \\
\hline & 0.6 & 0.7733 & 0.8843 & 0.8968 & 0.8447 & 0.9351 & 0.9479 & 0.9241 & 0.9793 & 0.9856 \\
\hline & 0.5 & 0.8006 & 0.8748 & 0.8980 & 0.8651 & 0.9303 & 0.9430 & 0.9381 & 0.9772 & 0.9853 \\
\hline \multirow[t]{6}{*}{0.50} & 1.0 & 0.8361 & 0.8928 & 0.9016 & 0.8949 & 0.9412 & 0.9459 & 0.9603 & 0.9851 & 0.9881 \\
\hline & 0.9 & 0.8491 & 0.8 & & & & & 70 & & 86 \\
\hline & 0.8 & 99 & 0.8852 & 0.8977 & 62 & 0.9 & 0.9 & 0.9 & 50 & 0.9874 \\
\hline & 0.7 & 727 & 0.8884 & 0.9031 & 0.9 & 0.9428 & 0.9525 & 0.9781 & 0.9865 & 0.9893 \\
\hline & 0.6 & 6 & 0.8 & 0.9 & 42 & 0.9478 & 0.9584 & 0.9 & 0.9879 & 0.9917 \\
\hline & 0.5 & 0.8675 & 0.8980 & 0.9167 & 0.9254 & 0.9538 & 0.9646 & 0.9749 & 0.9928 & 0.9944 \\
\hline \multirow[t]{6}{*}{0.75} & 1.0 & 0.8907 & 0.8959 & 0.9049 & 0.9444 & 0.9508 & 0.9565 & 0.9873 & 0.9908 & 0.9927 \\
\hline & 0.9 & 0.8853 & 0.8955 & 0.9050 & 0.9389 & 0.9492 & 0.9553 & 0.9861 & 0.9919 & 0.9940 \\
\hline & 0.8 & 0.8762 & 0.8999 & 0.9103 & 0.9304 & 0.9518 & 0.9573 & 0.9806 & 0.9925 & 0.9937 \\
\hline & 0.7 & 0.8544 & 0.9095 & 0.9163 & 0.9149 & 0.9604 & 0.9634 & 0.9718 & 0.9952 & 0.9951 \\
\hline & 0.6 & 0.8240 & 0.9176 & 0.9162 & 0.8867 & 0.9666 & 0.9629 & 0.9565 & 0.9949 & 0.9941 \\
\hline & 0.5 & 0.7700 & 0.9362 & 0.9216 & 0.8429 & 0.9761 & 0.9636 & 0.9283 & 0.9951 & 0.9923 \\
\hline \multirow[t]{6}{*}{0.95} & 1.0 & 0.8407 & 0.9034 & 0.9085 & 0.9018 & 0.9539 & 0.9551 & 0.9684 & 0.9919 & 0.9913 \\
\hline & 0.9 & 0.8097 & 0.9165 & 0.9118 & 0.8788 & 0.9662 & 0.9608 & 0.9556 & 0.9950 & 0.9933 \\
\hline & 0.8 & 0.7625 & 0.9184 & 0.9090 & 0.8375 & 0.9698 & 0.9596 & 0.9284 & 0.9949 & 0.9920 \\
\hline & 0.7 & 0.7187 & 0.9382 & 0.9189 & 0.8000 & 0.9766 & 0.9613 & 0.8998 & 0.9954 & 0.9909 \\
\hline & 0.6 & 0.6675 & 0.9501 & 0.9150 & 0.7521 & 0.9726 & 0.9489 & 0.8637 & 0.9923 & 0.9850 \\
\hline & 0.5 & 0.6173 & 0.9474 & 0.9032 & 0.6970 & 0.9702 & 0.9370 & 0.8169 & 0.9904 & 0.9781 \\
\hline
\end{tabular}


Table 6. A Comparison of AAN CI for $\alpha$ with that from Bain \& Engelhardt (BE) (1986)

\begin{tabular}{|c|c|c|c|c|c|c|c|c|c|c|}
\hline \multirow[b]{3}{*}{$n$} & \multirow[b]{3}{*}{$p_{0}$} & \multicolumn{5}{|c|}{ Coverage Probability } & \multicolumn{4}{|c|}{ Average Length } \\
\hline & & \multirow[b]{2}{*}{$\mathrm{CP}_{0}$} & \multicolumn{2}{|c|}{ AAN CI } & \multicolumn{2}{|c|}{ BE CI } & \multicolumn{2}{|c|}{ AAN CI } & \multicolumn{2}{|c|}{ BE CI } \\
\hline & & & MLE & MMLE & MLE & MMLE & MLE & MMLE & MLE & MMLE \\
\hline \multirow[t]{7}{*}{20} & 1.0 & 0.90 & 0.8748 & 0.9005 & 0.8569 & 0.8879 & 0.3848 & 0.4063 & 0.3938 & 0.4166 \\
\hline & 0.9 & & 0.8647 & 0.8957 & 0.8523 & 0.8847 & 0.4006 & 0.4373 & 0.4121 & 0.4510 \\
\hline & 0.8 & & 0.8567 & 0.8921 & 0.8421 & 0.8772 & 0.4046 & 0.4476 & 0.4168 & 0.4626 \\
\hline & 0.7 & & 0.8392 & 0.8814 & 0.8268 & 0.8632 & 0.4488 & 0.5260 & 0.4670 & 0.5506 \\
\hline & 0.6 & & 0.8491 & 0.8939 & 0.8360 & 0.8691 & 0.4866 & 0.5867 & 0.5117 & 0.6218 \\
\hline & 0.5 & & 0.8402 & 0.8955 & 0.8282 & 0.8539 & 0.5827 & 0.7638 & 0.6290 & 0.8366 \\
\hline & 0.4 & & 0.8353 & 0.9016 & 0.8145 & 0.8114 & 0.7945 & 1.2268 & 0.8933 & 1.4274 \\
\hline \multirow[t]{7}{*}{50} & 1.0 & & 0.8887 & 0.8991 & 0.8790 & 0.8940 & 0.2444 & 0.2494 & 0.2466 & 0.2517 \\
\hline & 0.9 & & 0.8892 & 0.9003 & 0.8816 & 0.8951 & 0.2509 & 0.2586 & 0.2535 & 0.2614 \\
\hline & 0.8 & & 0.8809 & 0.8936 & 0.8757 & 0.8885 & 0.2591 & 0.2690 & 0.2620 & 0.2722 \\
\hline & 0.7 & & 0.8778 & 0.8918 & 0.8723 & 0.8862 & 0.2815 & 0.2968 & 0.2855 & 0.3013 \\
\hline & 0.6 & & 0.8815 & 0.9008 & 0.8778 & 0.8903 & 0.3112 & 0.3330 & 0.3169 & 0.3395 \\
\hline & 0.5 & & 0.8747 & 0.8988 & 0.8757 & 0.8869 & 0.3687 & 0.4053 & 0.3787 & 0.4169 \\
\hline & 0.4 & & 0.8766 & 0.8977 & 0.8719 & 0.8777 & 0.4750 & 0.5462 & 0.4939 & 0.5699 \\
\hline \multirow[t]{7}{*}{20} & 1.0 & 0.95 & 0.9278 & 0.9443 & 0.9051 & 0.9314 & 0.4638 & 0.4901 & 0.4717 & 0.4995 \\
\hline & 0.9 & & 0.9203 & 0.9426 & 0.9002 & 0.9277 & 0.4829 & 0.5278 & 0.4921 & 0.5395 \\
\hline & 0.8 & & 0.9149 & 0.9412 & 0.8932 & 0.9234 & 0.4875 & 0.5401 & 0.4964 & 0.5520 \\
\hline & 0.7 & & 0.8954 & 0.9311 & 0.8800 & 0.9120 & 0.5426 & 0.6375 & 0.5556 & 0.6572 \\
\hline & 0.6 & & 0.9022 & 0.9368 & 0.8854 & 0.9125 & 0.5911 & 0.7151 & 0.6113 & 0.7463 \\
\hline & 0.5 & & 0.8959 & 0.9421 & 0.8786 & 0.8999 & 0.7187 & 0.9491 & 0.7624 & 1.0237 \\
\hline & 0.4 & & 0.8987 & 0.9513 & 0.8687 & 0.8626 & 1.0296 & 1.6294 & 1.1275 & 1.8513 \\
\hline \multirow[t]{7}{*}{50} & 1.0 & & 0.9417 & 0.9505 & 0.9318 & 0.9425 & 0.2926 & 0.2986 & 0.2945 & 0.3006 \\
\hline & 0.9 & & 0.9427 & 0.9496 & 0.9331 & 0.9450 & 0.3004 & 0.3096 & 0.3024 & 0.3118 \\
\hline & 0.8 & & 0.9344 & 0.9435 & 0.9252 & 0.9357 & 0.3101 & 0.3221 & 0.3123 & 0.3245 \\
\hline & 0.7 & & 0.9297 & 0.9405 & 0.9233 & 0.9349 & 0.3373 & 0.3558 & 0.3402 & 0.3592 \\
\hline & 0.6 & & 0.9348 & 0.9472 & 0.9305 & 0.9389 & 0.3735 & 0.3999 & 0.3782 & 0.4054 \\
\hline & 0.5 & & 0.9290 & 0.9 & 0.9289 & 0.9390 & 48 & 0.4893 & 0.4540 & 0.5004 \\
\hline & 0.4 & & 0.9294 & 0.9513 & 0.9258 & 0.9310 & 0.5805 & 0.6690 & 0.5985 & 0.6923 \\
\hline \multirow[t]{7}{*}{20} & 1.0 & 0.99 & 0.9751 & 0.9840 & 0.9553 & 0.9702 & 0.6272 & 0.6641 & 0.6203 & 0.6582 \\
\hline & 0.9 & & 0.9721 & 0.9830 & 0.9479 & 0.9649 & 0.6531 & 0.7159 & 0.6385 & 0.7023 \\
\hline & 0.8 & & 0.9704 & 0.9819 & 0.9423 & 0.9601 & 0.6588 & 0.7323 & 0.6369 & 0.7109 \\
\hline & 0.7 & & 0.9595 & 0.9767 & 0.9276 & 0.9466 & 0.7395 & 0.8741 & 0.7018 & 0.8358 \\
\hline & 0.6 & & 0.9597 & 0.9783 & 0.9310 & 0.9489 & 0.8156 & 0.9952 & 0.7756 & 0.9556 \\
\hline & 0.5 & & 0.9574 & 0.9801 & 0.9204 & 0.9329 & 1.0353 & 1.3945 & 0.9908 & 1.3562 \\
\hline & 0.4 & & 0.9637 & 0.9880 & 0.9019 & 0.8779 & 1.7702 & 3.0366 & 1.6014 & 2.7878 \\
\hline \multirow{7}{*}{50} & 1.0 & & 0.9851 & 0.9893 & 0.9791 & 0.9838 & 0.3890 & 0.3971 & 0.3874 & 0.3956 \\
\hline & 0.9 & & 0.9857 & 0.9890 & 0.9792 & 0.9847 & 0.3993 & 0.4117 & 0.3964 & 0.4089 \\
\hline & 0.8 & & 0.9821 & 0.9858 & 0.9742 & 0.9795 & 0.4124 & 0.4285 & 0.4081 & 0.4243 \\
\hline & 0.7 & & 0.9819 & 0.9863 & 0.9756 & 0.9804 & 0.4496 & 0.4747 & 0.4436 & 0.4688 \\
\hline & 0.6 & & 0.9805 & 0.9861 & 0.9765 & 0.9809 & 0.4999 & 0.5357 & 0.4941 & 0.5302 \\
\hline & 0.5 & & 0.9770 & 0.9856 & 0.9751 & 0.9809 & 0.6029 & 0.6647 & 0.5981 & 0.6606 \\
\hline & 0.4 & & 0.9784 & 0.9893 & 0.9750 & 0.9823 & 0.8155 & 0.9452 & 0.8061 & 0.9373 \\
\hline
\end{tabular}

Note: $\mathrm{CP}_{0}$ denotes the nominal coverage level. 
Table 7. A Summary of CIs for the Real Data Example

\begin{tabular}{|c|c|c|c|}
\hline & $90 \% \mathrm{CI}$ & $95 \% \mathrm{CI}$ & $99 \% \mathrm{CI}$ \\
\hline & \multicolumn{2}{|c|}{ CIs for $\alpha$} & \\
\hline Naive CI using MLE: & $(0.9020,1.5955)$ & $(0.8590,1.6963)$ & $(0.7830,1.9193)$ \\
\hline AAN CI using MLE: & $(0.9007,1.5994)$ & $(0.8576,1.7014)$ & $(0.7813,1.9276)$ \\
\hline AAN CI using MMLE: & $(0.8929,1.6412)$ & $(0.8477,1.7523)$ & $(0.7680,2.0002)$ \\
\hline \multirow[t]{2}{*}{ Wong-Wu Method: } & $(0.8958,1.6653)$ & $(0.8512,1.8000)$ & $(0.7637,2.1325)$ \\
\hline & \multicolumn{2}{|c|}{ CIs for 10 th percentile } & \\
\hline g MLE: & $(0.1163,0.2057)$ & $(0.1107,0.2187)$ & $(0.1009,0.2474)$ \\
\hline AAN CI u & $(0.0929,0.3603)$ & $(0.0857,0.4719)$ & $(0.0742,1.1642)$ \\
\hline AAN CI using MMLE: & $(0.0803,0.3381)$ & $(0.0738,0.4501)$ & $(0.0633,1.1721)$ \\
\hline \multirow[t]{2}{*}{ Wong-Wu Method: } & $(0.0663,0.2446)$ & $(0.0553,0.2666)$ & $(0.0370,0.3116)$ \\
\hline & \multicolumn{2}{|c|}{ CIs for 50th percentile } & \\
\hline Naive CI using MLE: & $(0.6461,1.1428)$ & $(0.6153,1.2150)$ & $(0.5608,1.3748)$ \\
\hline AAN CI using MLE: & $(0.6429,1.1522)$ & $(0.6118,1.2274)$ & $(0.5569,1.3950)$ \\
\hline AAN CI using MMLE: & $(0.6246,1.1593)$ & $(0.5927,1.2397)$ & $(0.5364,1.4197)$ \\
\hline \multirow[t]{2}{*}{ Wong-Wu Method: } & $(0.6176,1.1516)$ & $(0.5782,1.2302)$ & $(0.5036,1.4157)$ \\
\hline & \multicolumn{2}{|c|}{ CIs for 90th percentile } & \\
\hline Naive CI using MLE: & $(1.9273,3.4092)$ & $(1.8356,3.6246)$ & $(1.6730,4.1011)$ \\
\hline AAN CI using MLE: & $(1.7999,3.8624)$ & $(1.6965,4.2431)$ & $(1.5187,5.1891)$ \\
\hline AAN CI using MMLE: & $(1.8600,4.1784)$ & $(1.7470,4.6162)$ & $(1.5536,5.7139)$ \\
\hline \multirow[t]{2}{*}{ Wong-Wu Method: } & $(1.8801,4.2581)$ & $(1.7855,4.8006)$ & $(1.6267,6.2717)$ \\
\hline & \multicolumn{2}{|c|}{ CIs for MTTF } & \\
\hline Naive CI using MLE: & $(0.8735,1.5451)$ & $(0.8319,1.6427)$ & $(0.7582,1.8587)$ \\
\hline AAN CI using MLE: & $(0.8559,1.5992)$ & $(0.8126,1.7146)$ & $(0.7365,1.9777)$ \\
\hline AAN CI using MMLE: & $(0.8605,1.6834)$ & $(0.8140,1.8151)$ & $(0.7328,2.1183)$ \\
\hline Wong-Wu Method: & $(0.9011,1.9186)$ & $(0.8631,2.1157)$ & $(0.7941,2.6430)$ \\
\hline
\end{tabular}

